\title{
Prenatal stress and subsequent exposure to chronic mild stress influence dendritic spine density and morphology in the rat medial prefrontal cortex

\author{
Kimmo A Michelsen*1,2,3, Daniël LA van den Hove ${ }^{1,3}$, Christoph Schmitz ${ }^{1,3}$, \\ Olivier Segers ${ }^{1,3}$, Jos Prickaerts ${ }^{1,3}$ and Harry WM Steinbusch ${ }^{1,3}$
}

\begin{abstract}
Address: ${ }^{1}$ Department of Neuroscience, Faculty of Health, Medicine and Life Sciences, Maastricht University, P.O. Box 616, 6200 MD Maastricht The Netherlands, ${ }^{2}$ Department of Biology, Åbo Akademi University, Biocity, Tykistökatu 6 A, 20520, Turku, Finland and ${ }^{3}$ European Graduate School of Neuroscience (EURON)

Email: Kimmo A Michelsen* - k.michelsen@np.unimaas.nl; Daniël LA van den Hove - d.vandenhove@np.unimaas.nl;

Christoph Schmitz - c.schmitz@np.unimaas.nl; Olivier Segers - o.segers@bioch.unimaas.nl; Jos Prickaerts - jos.prickaerts@np.unimaas.nl ;

Harry WM Steinbusch - h.steinbusch@np.unimaas.nl

* Corresponding author
\end{abstract}

Published: 19 December 2007

BMC Neuroscience 2007, 8:107 doi:10.1186/147/-2202-8-107

This article is available from: http://www.biomedcentral.com//47/-2202/8//07

(C) 2007 Michelsen et al; licensee BioMed Central Ltd.

This is an Open Access article distributed under the terms of the Creative Commons Attribution License (http://creativecommons.org/licenses/by/2.0), which permits unrestricted use, distribution, and reproduction in any medium, provided the original work is properly cited.
Received: 14 March 2007

Accepted: 19 December 2007

\begin{abstract}
Background: Both prenatal stress (PS) and postnatal chronic mild stress (CMS) are associated with behavioral and mood disturbances in humans and rodents. The aim of this study was to reveal putative PS- and/or CMS-related changes in basal spine morphology and density of pyramidal neurons in the rat medial prefrontal cortex (mPFC).

Results: We show that rats exposed to PS and/or CMS display changes in the morphology and number of basal spines on pyramidal neurons in the mPFC. CMS had a negative effect on spine densities, particularly on spines of the mushroom type, which are considered to form stronger and more stable synapses than other spine types. PS alone did not affect spine densities, but had a negative effect on the ratio of mushroom spines. In addition, PS seemed to make rats less responsive to some of the negative effects of CMS, which supports the notion that PS represents a predictive adaptive response.
\end{abstract}

Conclusion: The observed changes may represent a morphological basis of PS- and CMS-related disturbances, and future studies in the field should not only consider total spine densities, but also separate between different spine types.

\section{Background}

An increasing amount of evidence indicates that exposure to prenatal stress (PS) increases the risk for developing psychopathology later in life [1]. In humans, PS has been associated with learning, behavioral and mood disorders, and rodent studies have linked PS to increased anxiogenic and depressive-like behavior and morphological and neu- rochemical changes in the brain $[2,3]$. Recent additions to the growing evidence include the demonstration of profound changes in dendritic arborization and spine densities in the rodent brain $[4,5]$. Exposure to chronic mild stress (CMS) in adulthood has also been linked to behavioral disturbances [6], as well as altered dendritic morphology [7]. 
The predictive adaptive response hypothesis states that the fetus responds to cues, which might be predictive of its future environment, and adapts its physiology accordingly. Thus, PS-related changes may provide a survival advantage if the offspring is born into a stressful environment. Some studies suggest that this may indeed be the case [8].

Of special interest in the psychopathology of PS and CMS is the medial prefrontal cortex (mPFC). The rat mPFC consists of a dorsal part, which includes the anterior cingulate cortex, and a ventral part [9] (Fig. 1). Both parts are implicated in executive function and, thus, the mPFC provides flexibility to affective processing $[10,11]$. Moreover, it has

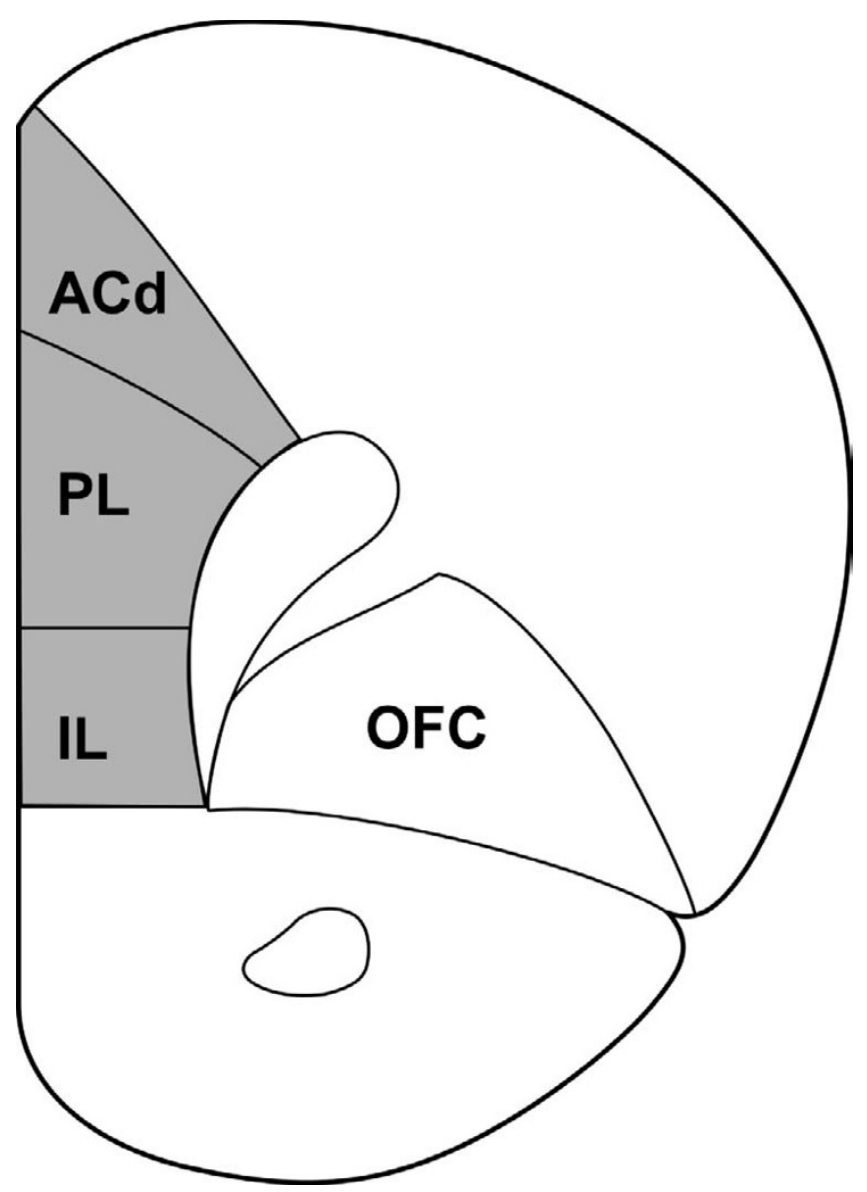

Figure I

A coronal section through the rat brain illustrating the mPFC (shaded area). The mPFC consists of a dorsal mPFC (dorsal anterior cingulate cortex and dorsal part of the prelimbic cortex) and a ventral mPFC (ventral part of the prelimbic cortex and infralimbic cortex) [9]. Neurons were filled in coronal sections at approximately $1.7 \mathrm{~mm}$ to $3.7 \mathrm{~mm}$ from bregma. ACd: dorsal anterior cingulated cortex; PL: prelimbic cortex; IL: infralimbic cortex; OFC: orbitofrontal cortex. Modified after [35]. recently been demonstrated that both the dorsal $[12,13]$ and ventral part $[14,15]$ of the mPFC determine how a stressor is controlled, at the level of brain structure activity as well as behavioral response. Thus, the mPFC may protect the subject against depression [16]. Along similar lines, the corresponding prefrontal areas in humans are decreased in volume [17], show a lower tissue organization $[18,19]$ and have specific changes in energy metabolism [20] in depression.

In the present study we evaluated whether PS and/or CMS exposure later in life leads to morphological changes at a basic functional level, i.e. basal dendritic spine density and morphology, in layer II and III pyramidal neurons of the rat $\mathrm{mPFC}$. The choice of layers was based on previous reports, which have shown stress-related changes in spine density in these layers $[5,15]$. For this purpose, we used a combination of intracellular iontophoretic injections with a fluorescent dye ("cell loading"), confocal microscopy and modern quantification software.

\section{Results}

Quantitative confocal microscopy analysis of the dendritic spines, imaged with the help of an intracellularly injected fluorescent dye, revealed PS- and/or CMS-related changes in spine number and/or morphology (Fig. 2). The measured parameters were: 1) Spine density, i.e. spine number per $\mu \mathrm{m}$ dendrite, which was expressed separately for each spine type (thin, mushroom, stubby) and for all types together (total spine density); 2) spine ratio, i.e. the abundance of each spine type relative to the total spine density; 3) the average thin spine length; 4) a combination of thin spine density and average thin spine length, i.e. thin spine length per $\mu \mathrm{m}$ dendrite. The purpose of this combined measure was to detect putative subtle thin spine dynamics, such as shrinkage, complete degeneration, or transformation to another spine type, which might go unnoticed when considering measures 2 and 3 separately. The results of the statistical analysis of spine type densities and ratios are presented in Table 1 and Fig. 3 , and of total spine density, thin spine length and thin spine length per $\mu \mathrm{m}$ dendrite in Table 2 and Fig. 3.

CMS had a clear negative effect on total spine density (Table 2 and Fig. 3) and on the density of spines of the mushroom type (Table 1 and Fig. 3), but not on the density of the two other spine types (Table 1 and Fig. 3). CMS did not have an effect on the average length of thin spines (Table 2 and Fig. 3). However, when average thin spine length and density were considered together, expressed as thin spine length per $\mu \mathrm{m}$ dendrite, CMS had a negative effect (Table 2 and Fig. 3). PS had a clear effect only on the ratio of mushroom spines (Table 1 and Fig. 3). 

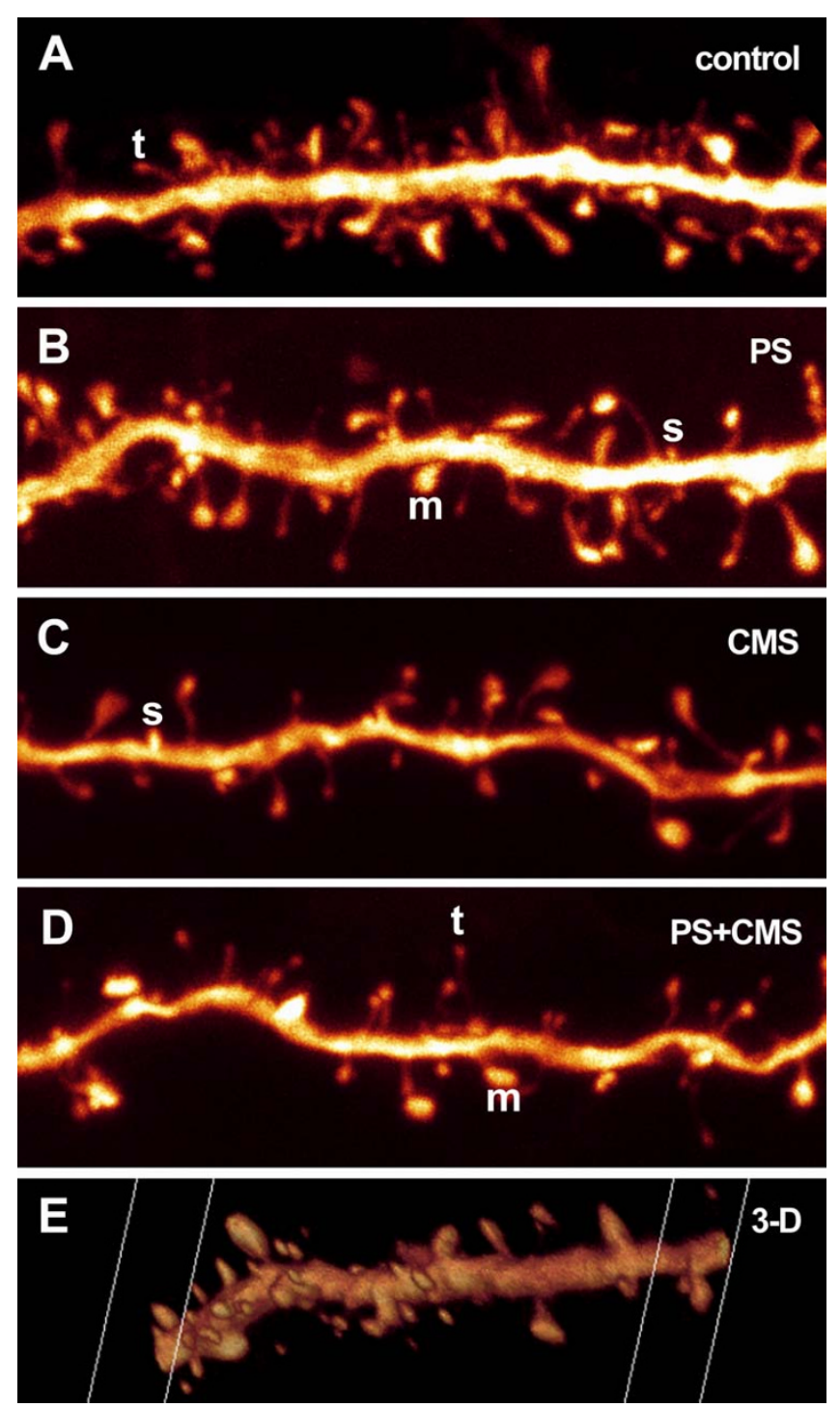

Figure 2

Maximum intensity projections of details from four of the stacks used for collection of spine data. The images are shown "raw" and have not undergone post-processing, such as contrast-enhancement (only rotation and resampling for screen-fit and printing purposes) (A-D). A screen shot of a three-dimensional (3-D) animation of one of the analyzed dendrites illustrates the 3-D advantage of the method $(E)$. The letters $t, m$ and $s$ exemplify thin, mushroom and stubby spines, respectively.

\section{Discussion}

CMS had a negative effect on the basal spine density of layer II and III pyramidal neurons in the left hemisphere of rat mPFC. This indicates that spines and, hence, probably also synaptic contacts are lost due to CMS. Spines of the mushroom type, characterized by a large spine head, were particularly affected. Spine head size correlates with post-synaptic density area and the number of presynaptic vesicles and has thus been suggested to reflect differences in synaptic efficacy [21]. Furthermore, large spines are more likely than small spines to contain smooth endoplasmic reticulum [22], which indicates differences in calcium-handling, as suggested [23]. In addition, mushroom spines seem to form more stable synapses than thin spines, which have higher motility and form more transient contacts [24]. Thus, it can be speculated that the loss of mushroom spines has a more profound effect on neuron function than the loss of the other types does, and than what could be expected if only the loss of total spine numbers were considered. However, it must be kept in mind that some synapses may turn into shaft synapses, as the spines are lost, and that shaft synapses cannot be quantified with the method we used.

Spines are dynamic and may change shape and size, as well as appear or disappear altogether [23]. In an attempt to evaluate whether spines were shrinking or growing due to PS and/or CMS, we measured the lengths of the thin spines (the other two types were not measured because due to their small or absent necks the results would have been too imprecise). Somewhat surprisingly, the average length of the spines was neither affected by CMS, nor PS nor by their combination. However, when the average length of thin spines was considered in combination with the density of thin spines (which showed a decreasing CMS-dependent trend) a negative CMS-effect was observed. This effect could possibly be attributed to putative conformational changes of the thin spines, as they are in the process of either transforming into another spine type or disappearing completely.

Our results show, that CMS leads to dendritic spine loss in the medial prefrontal cortex of the left hemisphere. Furthermore, PS seems to decrease the vulnerability to some of the degenerating effects of CMS. It is tempting to speculate that this is due to a predictive adaptive response, although it must be kept in mind that the current study does not provide convincing evidence for it. Nevertheless, it has been shown, that the fetal environment can influence the risk of postnatal disease and the ability to cope with the postnatal environment [25] and, indeed the difference in thin spine length per $\mu \mathrm{m}$ dendrite was significantly decreased in the CMS group as compared to controls, but not in the CMS+PS group, whose average (1.591) was close to that of the PS group (1.648). It should be noted, however, that the values for the CMS+PS group were not reversed to control levels, which could be expected of a strong predictive adaptive response. In addition, a trend towards an interaction between PS and CMS was observed with regard to spine length per $\mu \mathrm{m}$ dendrite and thin spine ratio and stubby spine ratio, which might reflect the observed putative compensatory effect of PS on CMS. The notion that PS could represent a predictive 
Table I: Statistical analysis

\begin{tabular}{|c|c|c|c|c|c|c|c|}
\hline & \multirow[t]{2}{*}{ Spine type: } & \multicolumn{2}{|c|}{ Thin } & \multicolumn{2}{|c|}{ Mushroom } & \multicolumn{2}{|c|}{ Stubby } \\
\hline & & $F$ value & $P$ value & F value & $P$ value & F value & $P$ value \\
\hline \multirow[t]{3}{*}{ Spine density } & PS effect & 0.17 & 0.686 & 3.67 & 0.079 & 0.04 & 0.848 \\
\hline & CMS effect & 3.56 & 0.084 & 5.33 & $\underline{0.040}$ & 3.63 & 0.081 \\
\hline & $\begin{array}{l}\text { PSxCMS } \\
\text { interaction }\end{array}$ & 2.56 & 0.136 & 2.16 & 0.167 & 0.36 & 0.562 \\
\hline \multirow[t]{3}{*}{ Spine ratio } & PS effect & 3.52 & 0.085 & 5.92 & $\underline{0.032}$ & 0.13 & 0.730 \\
\hline & CMS effect & 0.89 & 0.365 & 0.22 & $\overline{0.647}$ & 0.34 & 0.573 \\
\hline & $\begin{array}{l}\text { PSxCMS } \\
\text { interaction }\end{array}$ & 3.65 & 0.080 & 0.15 & 0.707 & 3.79 & 0.075 \\
\hline
\end{tabular}

Results of statistical analysis ( $F$ and $P$ values) with generalized linear model MANOVA for each spine type separately.

adaptive response, which makes rats less responsive, but not unresponsive, to the negative effects of CMS, is further supported by our observation that, when subjected to the home cage emergence test, CMS rats showed signs of increased anxiety. This effect was not observed in CMS rats, which had previously been exposed to PS (unpublished observation).

No effect of PS on spine density was observed. However, the negative effect of PS on the ratio of mushroom spines suggests that PS may induce some spine rearrangements in the neurons studied. The result becomes more interesting with regard to future studies when one considers the suggested relatively strong synaptic strength of mushroom spines, and the fact that PS showed a trend towards a decrease in mushroom spine density. Various studies have demonstrated a decrease in, for example, synaptophysin immunoreactivity, after PS [2,3]. In line with the fact that mushroom spines have a larger postsynaptic density and more synaptic vesicles than smaller spines do [21], quantification of immunoreactivity for postsynaptic density and synaptic vesicle markers in relation to synaptophysin immunoreactivity could further elucidate the dynamics of spines in the brain of rats exposed to PS.

In this study, we divided the spines into the three main categories introduced by Peters and Kaiserman-Abramof in 1970 [26]. However, in reality the various spine shapes fall along a continuum of different neck lengths and head sizes; even branched spines exist; see [23]. For example, Garcia-Lopez and coworkers used a classification into six types [27]. A more detailed analysis of spine morphology in combination with new software, which allows rapid and automated quantification of spine numbers and shapes, is likely to give new insights to spine dynamics in the near future; see [28] for some of the latest methodological advances.
A recent study by Murmu and coworkers [5] showed that PS correlates with changes in spine density and dendritic tree arborization in dorsal anterior cingulate (ACd) and orbitofrontal cortex (OFC). That study did not find a clear difference in total spine densities of the basal dendrites of ACd pyramidal neurons in male PS versus male control animals. The finding is in agreement with our study, which included the ACd in the mPFC area. Furthermore, Murmu and coworkers found that the apical dendrite spine density was decreased in male PS rats. We did not measure basal dendritic length, but they found no effect of PS on this measure. Along similar lines, several studies have reported that chronic stress in male rats only affects the length of apical dendrites but not of basal ones [7,2931]. This suggests that it is feasible to assume that in our study basal dendrite arborization would not have been affected in any of the experimental groups. Further, chronic restraint stress has been shown to affect apical dendritic spine densities in the MPFC, whereas basal dendritic spines were not affected [32]. This is in contrast to our finding, which showed that spine densities on the basal tree were indeed affected. The discrepancy is probably due to the difference in stress (chronic restraint stress vs. variable CMS) and our inclusion of the infralimbic cortex.

Murmu and coworkers also studied the OFC after PS and found that spine densities in male rats were decreased both on apical and basal dendrites. In female rats, spine densities were decreased in both dendrite types in both ACd and OFC. In all cases of spine reductions in both females and males, this reduction was approximately $20 \%$ [5]. Yet, dendritic length was not affected in these brain areas of female rats after PS.

One can argue, that the behavioral testing of the rats used in this study may have affected spine morphology and 

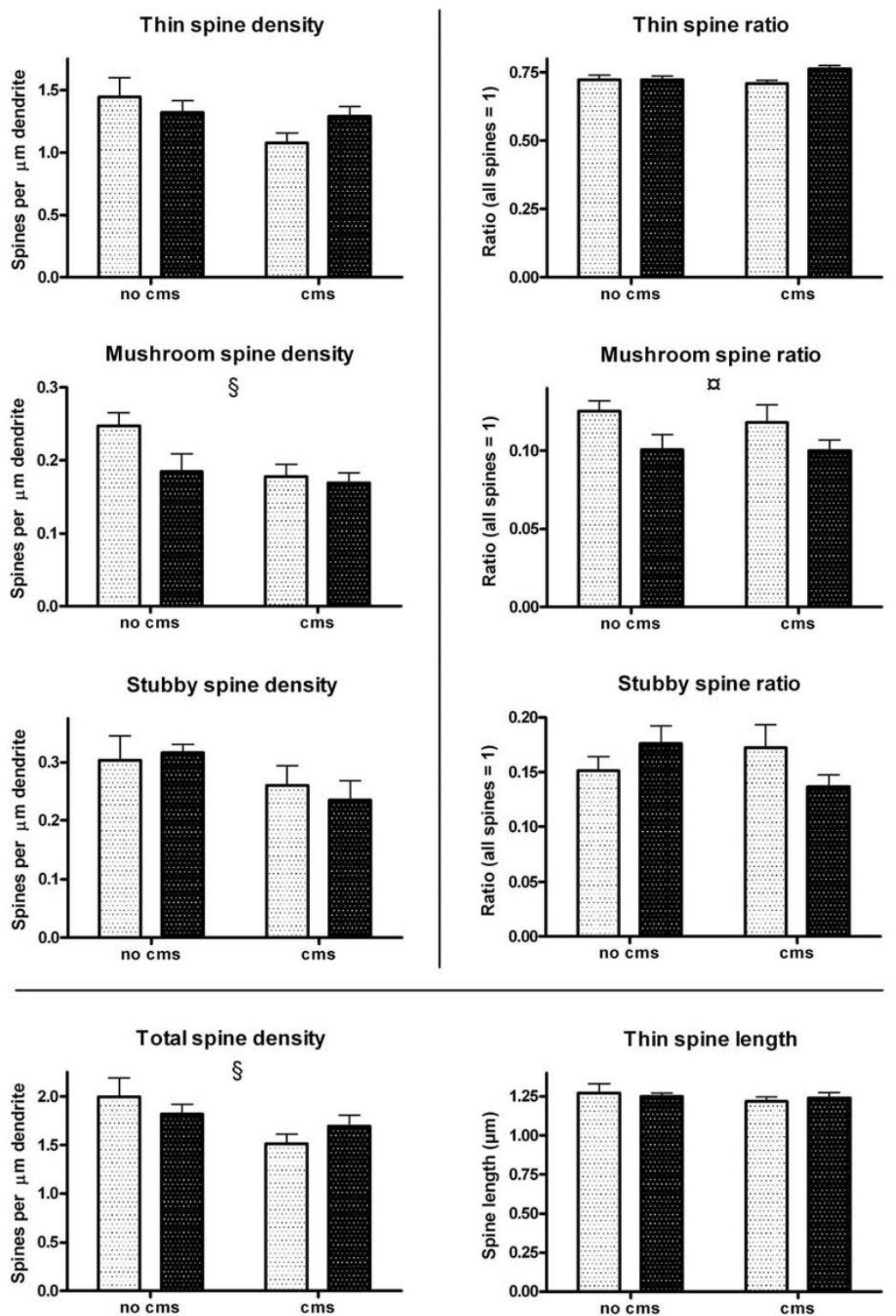

Thin spine length

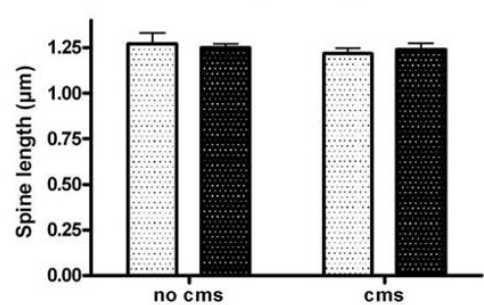

Thin spine length per $\mu \mathrm{m}$ dendrite

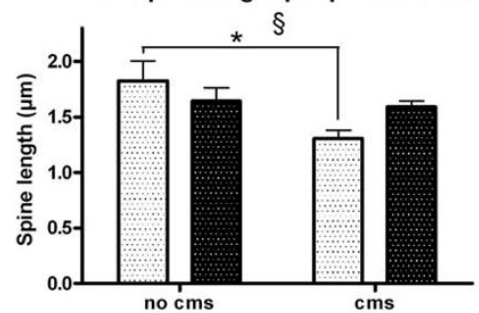

Figure 3

The densities (left column) and ratios (right column) of each spine type separately. The total density of spines, average length of thin spines and thin spine length per $\mu \mathrm{m}$ dendrite are shown at the bottom. Light columns = no PS; dark columns = PS. Error bars show SEM. § CMS effect $(\mathrm{P}<0.05)$; : $\mathrm{PS}$ effect $(\mathrm{P}<0.05)$; *: $\mathrm{P}<0.05$ in Bonferroni post-hoc analysis. See also tables I and 2. 
Table 2: Statistical analysis

\begin{tabular}{|c|c|c|c|c|c|c|}
\hline & \multicolumn{2}{|c|}{ Total spine density } & \multicolumn{2}{|c|}{ Thin spine length } & \multicolumn{2}{|c|}{$\begin{array}{l}\text { Thin spine length/ } \\
\mu \mathrm{m} \text { dendrite }\end{array}$} \\
\hline & F value & $P$ value & $\mathrm{F}$ value & $P$ value & F value & $P$ value \\
\hline PS effect & 0.00 & 0.988 & 0.00 & 0.965 & 0.23 & 0.644 \\
\hline CMS effect & 5.26 & $\underline{0.041}$ & 0.67 & 0.431 & 6.03 & $\underline{0.030}$ \\
\hline $\begin{array}{l}\text { PSxCMS } \\
\text { interaction }\end{array}$ & 1.73 & $\overline{0.207}$ & 0.32 & 0.584 & 3.89 & $\overline{0.072}$ \\
\hline
\end{tabular}

Results of statistical analysis ( $F$ and $P$ values) with ANOVA for the total density of spines, average length of thin spines and thin spine length per $\mu \mathrm{m}$ dendrite.

numbers, but it has to be noted that rats were left undisturbed for two weeks after testing and testing itself did not include any chronic stressful events including administration of repeated food chocks or chronic restraint which is known to result in long-lasting structural changes in spines [33]. Furthermore, since all animals underwent exactly the same testing procedure, it is unlikely that the behavioral testing would be responsible for the significant effects of PS and/or CMS on spines presented here.

Due to limitations imposed by the fact that the rats, which were used for this experiment, were also used to study other, as yet unpublished, putative effects of PS and/or CMS, we decided to concentrate our efforts on basal spines only. For example, the animals had to be killed within a small time-window in order to exclude the possibility that different survival times after the behavioral experiments would affect the spine data. Cell loading is time-consuming and must be done within a matter of days after perfusion of the brain, so the time-restraint prevented us from loading enough neurons to be able to include both dendrite types in the analysis. With the current results at hand, it is evident that future experiments on the effects of PS and/or CMS on spine number and morphology could benefit from including both apical and basal dendrites. Studies involving the mPFC could also benefit from taking into account the heterogenicity of the mPFC, instead of treating it as one entity, in order to minimize bias introduced by possible differences in sampling within the chosen area, and to detect possible differences between the parts of the mPFC. In addition, they could benefit from analysing a larger number of animals per group than was done in this study, in order to provide more convincing statistical evidence of putative effects.

With these suggested improvements for future studies, we acknowledge the methodological limitations of the current one. Nevertheless, we present statistically significant results on the effects of PS and/or CMS on dendritic spines in the MPFC, which should encourage further, more detailed, studies on PS and/or CMS-related effects on the brain.

The method of cell loading (also called cell filling) in combination with laser confocal microscopy offers several clear advantages to, for instance, the traditional method of analyzing Golgi-preparations under an epifluorescence microscope: 1) Injecting a fluorescent dye into a single neuron makes it possible to analyze that neuron without interference from nearby dendrites. 2) Injection can be done at random, whereas it is not clear why the Golgimethod stains some neurons and leaves others unstained. 3) Laser confocal analysis of loaded dendrites and spines allows three-dimensional analysis, so that spines immediately below or above the dendrite can be distinguished. 4) The high resolution reveals spines, which go unnoticed in regular fluorescence microscopy, and allows one to distinguish different types of spines, as demonstrated here.

\section{Conclusion}

In summary, this study is the first to show CMS-dependent morphological changes on the level of basal dendritic spines in the rat MPFC, and PS seems to make the brain less responsive to some of the stress-related changes as implicated by the predictive adaptive response hypothesis.

\section{Methods \\ Animals}

This experiment was approved by the Animal Ethics Board of the University of Maastricht, the Netherlands. Acclimatized pregnant Sprague-Dawley rats (Charles River, The Netherlands) were housed individually within a temperature-controlled environment $\left(21 \pm 1{ }^{\circ} \mathrm{C}\right)$ with a $1: 1$ light:dark cycle (lights on at 7.00) and had access to food and water ad libitum.

Restraint stress was performed daily during the last week of pregnancy (embryonic day 14-21). Pregnant rats were restrained three times a day for $45 \mathrm{~min}$ in transparent 
plastic cylinders and simultaneously exposed to bright light, as described [34]. Female rats from the control group were left undisturbed in their home cage.

At postnatal day 21 (P21), male pups ( $\mathrm{n}=8$ /group) were weaned and housed together (2 rats/cage) and kept at a reversed day-night cycle from this point onward (lights on at 17.00) in order to make it practically feasible to perform behavioral experiments. At P77, 4 animals from each of the 2 groups were subjected to variable CMS for three weeks, resulting in the four following groups $(n=4$ / group): control (untreated), PS, CMS and PS+CMS. Stressors (housing in mice cage, cage tilt [angle of $45^{\circ}$ ], housing in an empty cage [no sawdust], wet bedding in cage [200 $\mathrm{ml}$ cold water added per cage], flashing light [stroboscope; low intensity, $2.5 \mathrm{~Hz}$ ) during the dark phase were applied in a random order. Two stressors per day, each lasting for three hours, were applied. Subsequently, the animals were subjected to various behavioral tasks, after which they were left undisturbed for approximately 2 weeks and used for the current study at P100.

\section{Tissue preparation}

The rats were perfused transcardially with $1 \%$ paraformaldehyde for 1 min followed by a mixture of $4 \%$ paraformaldehyde and $0.125 \%$ glutaraldehyde in $0.1 \mathrm{M}$ phosphate buffer, pH 7.4 (PB) for $11 \mathrm{~min}$. The brain was removed and hemisected. The left hemisphere was postfixed for $4 \mathrm{~h}$ in the same mixture and then moved to $\mathrm{PB}$ with $0.02 \% \mathrm{NaN}_{3}$ to await sectioning at $200 \mu \mathrm{m}$ on a vibratome. The right hemisphere was used in another study.

\section{Cell loading}

The sections were incubated with Hoechst (in PB) for at least $15 \mathrm{~min}$ to reveal the cell nuclei under UV illumination. They were then rinsed in $\mathrm{PB}$, mounted on a nitrocellulose filter and immersed in $\mathrm{PB}$ under a fluorescence microscope (Leica DMLFS; Leica Microsystems, Heidelberg, Germany) equipped with a $40 \times$ water immersion objective. Using a glass micropipette attached to a micromanipulator (MP-85; Sutter instrument, Novato, CA, USA) a fluorescent dye (Lucifer Yellow $\mathrm{CH}$ lithium salt; Invitrogen, Carlsbad, CA, USA) was injected under a direct current of 1-6 nA for 6-12 min (current source: Model 260, World Precision Instruments, Sarasota, FL, USA) into layer II and III pyramidal neurons in the medial prefrontal cortex until the dye had reached the distal dendrites and no further loading was observed. The neurons were chosen in a systematic manner at approximately equal distances from each other, in an attempt to obtain an equal representation of subregions. The loaded neurons were sufficiently far apart for their dendritic trees not to overlap.

\section{Imaging}

Three-dimensional image stacks of basal dendrites were collected with a Leica TCS-SP confocal laser scanning microscope system equipped with an Ar-Kr laser (Melles Griot, Carlsbad, CA, USA). LY was excited with the 488 $\mathrm{nm}$ laser line and emission was collected in the 490-690 $\mathrm{nm}$ interval. Confocal microscopy image stacks with an xy pixel size of $0.05 \times 0.05 \mu \mathrm{m}$ and a step-size of $0.2 \mu \mathrm{m}$ between optical planes were acquired with a $100 \times$ n.a. 1.4 oil immersion objective with a theoretical lateral and axial resolution of 0.136 and $0.291 \mu \mathrm{m}$, respectively. Up to 3 stacks per neuron were collected at randomly selected sites at a radial distance of 25-105 $\mu \mathrm{m}$ from the soma. The average dendrite length per site was $33 \mu \mathrm{m}$, so the length per cell was up to $100 \mu \mathrm{m}$. Branch order was not addressed. The samples were first divided into proximal (25-55 $\mu \mathrm{m}$ from soma), medial (50-80 $\mu \mathrm{m}$ from soma) and distal (75-105 $\mu \mathrm{m}$ from soma) but were later merged because no significant differences could be seen between the three divisions.

\section{Spine quantification and measurements}

The image stacks were opened in Neurolucida software (Microbrightfield, Williston, VT, USA) and spines were counted and labeled as thin, stubby or mushroom type based on morphology as follows: thin spines = long narrow necks and small to medium-sized heads; mushroom spines $=$ short necks and big heads; stubby spines $=$ short protrusion with no clear necks (Fig. 2). The length of the thin spines was measured with the same software. A total of 7426 spines were counted in 4-6 neurons/rat and 4 rats/group.

\section{Statistics}

The effects of CMS and PS on the densities and ratios of thin, mushroom and stubby spines were evaluated using MANOVA. The effects on total spine density, average spine length and spine length per $\mu \mathrm{m}$ dendrite were evaluated using ANOVA (prenatal condition x postnatal condition) and analyzed in more detail using Bonferroni post-hoc tests. Statistical significance was defined as $\mathrm{P}<$ 0.05. All statistics were carried out using SPSS software version 14 (SPSS Inc, Chicago, IL, USA).

\section{Authors' contributions}

KAM contributed to the design of the study, participated in the tissue preparation, performed the cell loading, confocal imaging, spine quantification and data collection, contributed to the statistical analysis and drafted the manuscript. DLAvdH contributed to the design of the study, performed the PS and CMS treatment and maintained the rats, contributed significantly to the statistical analysis and assisted substantially with drafting the manuscript. CS assisted with drafting the manuscript. OS assisted with the cell loading and performed the spine length measure- 
ments. JP contributed to the design of the study, contributed significantly to the PS and CMS treatment, maintenance of the rats and the statistical analysis and assisted substantially with drafting the manuscript. HWMS contributed to the design of the study and assisted with drafting the manuscript. All authors approved the manuscript.

\section{Acknowledgements}

The authors wish to thank Hellen Steinbusch for extensive help in the lab. KAM, DLAvdH and JP were supported by European Union Framework 6 Integrated Project NEWMOOD Grant LSHM-CT-2004-503474. KAM was also supported by grants from Helsingin Sanomain 100-vuotissäätiö, Alfred Kordelinin yleinen edistys- ja sivistysrahasto, Orionin tutkimussäätiö and K. Albin Johanssons stiftelse.

\section{References}

I. Huizink AC, Mulder EJ, Buitelaar JK: Prenatal stress and risk for psychopathology: specific effects or induction of general susceptibility? Psychol Bull 2004, I30: | |5-|42.

2. Weinstock $M$ : Alterations induced by gestational stress in brain morphology and behaviour of the offspring. Prog Neurobiol 200I, 65:427-45I

3. Weinstock $M$ : The potential influence of maternal stress hormones on development and mental health of the offspring. Brain Behav Immun 2005, 19:296-308.

4. Ishiwata $H$, Shiga T, Okado N: Selective serotonin reuptake inhibitor treatment of early postnatal mice reverses their prenatal stress-induced brain dysfunction. Neuroscience 2005, | 33:893-90|.

5. Murmu MS, Salomon S, Biala Y, Weinstock M, Braun K, Bock J Changes of spine density and dendritic complexity in the prefrontal cortex in offspring of mothers exposed to stress during pregnancy. Eur J Neurosci 2006, 24:|477-|487.

6. Willner P: Chronic mild stress (CMS) revisited: consistency and behavioural-neurobiological concordance in the effects of CMS. Neuropsychobiology 2005, 52:90-II0.

7. Brown SM, Henning S, Wellman CL: Mild, short-term stress alters dendritic morphology in rat medial prefrontal cortex. Cereb Cortex 2005, I 5: I714-1722.

8. Gluckman PD, Hanson MA: Adult disease: echoes of the past Eur J Endocrinol 2006, I 55 Suppl I:S47-S50.

9. Heidbreder CA, Groenewegen HJ: The medial prefrontal cortex in the rat: evidence for a dorso-ventral distinction based upon functional and anatomical characteristics. Neurosci Biobehav Rev 2003, 27:555-579.

10. Bush G, Luu P, Posner MI: Cognitive and emotional influences in anterior cingulate cortex. Trends Cogn Sci 2000, 4:215-222.

II. Davidson RJ: Anxiety and affective style: role of prefrontal cortex and amygdala. Biol Psychiatry 2002, 5 I:68-80.

12. Bissiere S, McAllister KH, Olpe HR, Cryan JF: The rostral anterior cingulate cortex modulates depression but not anxietyrelated behaviour in the rat. Behav Brain Res 2006, I75:195-199.

13. Drevets WC: Prefrontal cortical-amygdalar metabolism in major depression. Ann N Y Acad Sci 1999, 877:6| 4-637.

14. Amat J, Baratta MV, Paul E, Bland ST, Watkins LR, Maier SF: Medial prefrontal cortex determines how stressor controllability affects behavior and dorsal raphe nucleus. Nat Neurosci 2005 8:365-37I.

15. Radley J, Arias CM, Sawchenko PE: Regional differentiation of the medial prefrontal cortex in regulating adaptive responses to acute emotional stress. J Neurosci 2006, 26:12967-12976.

16. Robbins TW: Controlling stress: how the brain protects itself from depression. Nat Neurosci 2005, 8:26I-262.

17. Drevets WC: Neuroimaging and neuropathological studies of depression: implications for the cognitive-emotional features of mood disorders. Curr Opin Neurobiol 200 I, I I :240-249.

18. Bae JN, MacFall JR, Krishnan KR, Payne ME, Steffens DC, Taylor WD: Dorsolateral prefrontal cortex and anterior cingulate cortex white matter alterations in late-life depression. Biol Psychiatry 2006, 60:1356-1363

19. Drevets WC, Price JL, Simpson JR Jr., Todd RD, Reich T, Vannier M, Raichle ME: Subgenual prefrontal cortex abnormalities in mood disorders. Nature 1997, 386:824-827.

20. Mayberg HS, Lozano AM, Voon V, McNeely HE, Seminowicz D, Hamani C, Schwalb JM, Kennedy SH: Deep brain stimulation for treatment-resistant depression. Neuron 2005, 45:65I-660.

2I. Harris KM, Stevens JK: Dendritic spines of CA I pyramidal cells in the rat hippocampus: serial electron microscopy with reference to their biophysical characteristics. J Neurosci 1989, 9:2982-2997.

22. Spacek J, Harris KM: Three-dimensional organization of smooth endoplasmic reticulum in hippocampal CAI dendrites and dendritic spines of the immature and mature rat. J Neurosci 1997, I 7:190-203.

23. Nimchinsky EA, Sabatini BL, Svoboda K: Structure and function of dendritic spines. Annu Rev Physiol 2002, 64:3 I3-353.

24. Konur S, Yuste R: Imaging the motility of dendritic protrusions and axon terminals: roles in axon sampling and synaptic competition. Mol Cell Neurosci 2004, 27:427-440.

25. Gluckman PD, Hanson MA: Developmental origins of disease paradigm: a mechanistic and evolutionary perspective. Pediatr Res 2004, 56:3 I I-317.

26. Peters A, Kaiserman-Abramof IR: The small pyramidal neuron of the rat cerebral cortex. The perikaryon, dendrites and spines. Am J Anat 1970, 127:321-355.

27. Garcia-Lopez P, Garcia-Marin V, Freire M: Three-dimensional reconstruction and quantitative study of a pyramidal cell of a Cajal histological preparation. $\int$ Neurosci 2006, 26: I I249- I I 252.

28. Wearne SL, Rodriguez A, Ehlenberger DB, Rocher AB, Henderson $S C$, Hof PR: New techniques for imaging, digitization and analysis of three-dimensional neural morphology on multiple scales. Neuroscience 2005, 136:661-680.

29. Cook SC, Wellman CL: Chronic stress alters dendritic morphology in rat medial prefrontal cortex. I Neurobiol 2004, 60:236-248

30. Liston C, Miller MM, Goldwater DS, Radley J], Rocher AB, Hof PR, Morrison JH, McEwen BS: Stress-induced alterations in prefrontal cortical dendritic morphology predict selective impairments in perceptual attentional set-shifting. J Neurosci 2006 , 26:7870-7874.

31. Radley JJ, Rocher AB, Janssen WG, Hof PR, McEwen BS, Morrison JH: Reversibility of apical dendritic retraction in the rat medial prefrontal cortex following repeated stress. Exp Neurol 2005, 196:199-203.

32. Radley J, Rocher AB, Miller M, Janssen WG, Liston C, Hof PR, McEwen $\mathrm{BS}$, Morrison JH: Repeated stress induces dendritic spine loss in the rat medial prefrontal cortex. Cereb Cortex 2006, 16:313-320.

33. Mitra R, Jadhav S, McEwen BS, Vyas A, Chattarji S: Stress duration modulates the spatiotemporal patterns of spine formation in the basolateral amygdala. Proc Natl Acad Sci U S A 2005, I 02:937|-9376.

34. Ward IL, Weisz ]: Differential effects of maternal stress on circulating levels of corticosterone, progesterone, and testosterone in male and female rat fetuses and their mothers. Endocrinology 1984, I | 4: I 635- I 644.

35. Paxinos G, Watson C: The Rat Brain in Stereotaxic Coordinates San Diego, CA, Academic Press; 1997. 